\title{
Putting people and robots together in manufacturing: are we ready?
}

\author{
S.R. Fletcher ${ }^{*}$, T.L. Johnson $†$ and J. Larreina \\ $\uparrow$ Centre for Structures, Assembly and Intelligent Automation, Cranfield University, UK \\ + Industrial Management and Innovation Unit, IK4-Tekniker, Spain \\ * Corresponding author, email: s.fletcher@cranfield.ac.uk
}

\begin{abstract}
Traditionally, industrial robots have been completely segregated from people in manufacturing systems to mitigate the dangers posed by their operational speeds and heavy payloads. Putting human operators together with large-scale industrial robots is now becoming increasingly possible with the development of integrated safety monitoring systems, and with smaller force-limited robotics that are now being produced with sufficient robustness for industry. However, with long-standing perceptions of robots as hazardous, we do not yet know how manufacturing workforces will accept collaborative systems with either large or small scale robotics and there is a need to identify and define new ethical and safety standard requirements for integrating people and robots to work collaboratively in industrial assembly tasks. To date there is little or no attention to ethical issues or psychological safety in the industrial safety standards that govern robotics and automated work systems. This paper describes the current situation and specific ways in which human-robot collaboration will significantly improve efficiency and flexibility, and outlines some early work that is being performed to identify the requirements that will be needed in order to facilitate this new way of bringing people and robots together in manufacturing. It presents a brief summary of initial findings that support the need for ethical issues to be considered as a candidate for new and / or revised safety standards.
\end{abstract}

Keywords: advanced manufacturing technology, industrial robots, human-robot collaboration, industrial safety

\section{INTRODUCTION}

The manufacturing industry is currently being revolutionised by digitisation and automation. As part of this movement, organisations are pushing hard to escalate the development and application of industrial robotics in factories. The International Federation of Robotics predicts that by 2019 there will be 2.5 million industrial robots in production systems around the world, reflecting a $12 \%$ average annual growth rate [1].

Traditional large, high payload industrial robots have in the past presented such a significant hazard to humans that it has been necessary to keep them completely segregated from workers, within fully automated stations behind physical guarding and safe-separation measures (e.g. fencing, barriers, laser curtains, etc.). Full automation of a production process is rarely feasible as, in most cases there are assembly tasks that require human dexterity and cognitive reasoning. Therefore, hybrid systems have tended to locate industrial robots upstream in enclosed zones for the performance of simple and repetitive tasks, and locate operators in separate areas downstream in the system to perform more complex and varied assembly tasks [2]. As these arrangements and boundaries have been customary for a long period of time, operators have long been aware of the potential risk posed by industrial robots and the safety requirement for them to remain at a safe distance from robot operating zones.

In more recent years, advances in sensor based safety control functions along with some concomitant changes in safety standards have for some time now made it possible, within predefined specifications, to remove the traditional safe separation boundaries needed for heavy industrial robots and allow people and robots to work more closely together in shared spaces [3]. In addition, advances in technology have increased the development and availability of smaller, lighter force-limited robots which are specifically designed for collaboration with people and highly applicable for joint performance of assembly tasks [4, 5]. Together, these fast-developing capabilities bring a new concept of industrial human-robot collaboration (HRC) which offers the manufacturing industry substantial benefits for enhancing production efficiency and flexibility. The question is: are we ready in terms of understanding what is now needed in robot ethics and safety standards?

This paper summarises the practical benefits of developing HRC solutions and describes current research work which is identifying requirements 
and, at the same time, unearthing where current ethics and safety standards do not adequately meet the needs of future systems. The main purpose of the paper is to illustrate the need for greater consideration and acceptance of ethical and usercentred principles in new or revised safety standards for collaborative robotics in the manufacturing industry.

\section{COLLABORATIVE INDUSTRIAL ROBOT SOLUTIONS}

The rise of $\mathrm{HRC}$ in manufacturing facilities is expected to provide a number of tangible improvements to the efficiency and flexibility of modern production systems.

\subsection{Efficiency}

HRC will enable improvements to manufacturing efficiency via two key related developments: more expedient co-location and more suitable human/robot function allocation.

Firstly, the traditional need to physically separate automated and manual processes has been disruptive to system continuity and inhibits batch production flexibility [6]. Shared-space HRC solutions that co-locate humans and robots will enable better synchronisation and sequencing to make work flow more efficient whilst also maintaining human skills and employment [7].

Second, the traditional need to segregate industrial robots in designated zones has meant that people have had to continue to perform many unhealthy or mundane manual tasks which would be more suited to robotics in work areas outside of these protected zones. As HRC will allow human operators and robots to co-exist in shared workspaces this will enable more suitable and balanced allocation of task functions that better exploits and complements the strengths of both human and robot skills in assembly work. This means that industrial robotics will not replace human skills but will relieve people from alienating and potentially injurious tasks, and provide opportunities for them to contribute more "valueadded work" [8].

\subsection{Flexibility}

HRC will also help organisations to address two key requirements for flexibility in modern times: system responsiveness and workforce skills fluidity.

Firstly, there is a growing need for production systems to be more responsive and adaptable to fluctuating consumer demands for personalised products. Mass customisation means large scale production of a wider variety of product variants but in smaller batch sizes without compromising "cost, delivery and quality” [9]. HRC systems provide the increased intelligence and flexibility that helps lower the cost and feasibility of this required degree of reconfigurability [10].

Second, many years of globalisation and various demographic / social transitions have led to a changing and more fluid complexion of workforces due to escalating workforce mobility (skilled and unskilled) [11], ageing populations and extended working lives [12], greater social demands for workplace inclusivity of diversity [13]. These evolving trends bring a wider, more diverse and transient set of worker capabilities and skills that manufacturing organisations will need to be able to accommodate. As HRC solutions offer improved reconfigurability and reallocation of tasks between people and robots they provide a way in which systems can be designed and redesigned to 'bridge gaps in skills' [14]. In theory, HRC should therefore not only provide a means of accommodating more adaptiveness to meet changing production requirements, but also to suit the personal needs of workers and their various cultural and idiosyncratic differences - ideally without the need for too much (re)training.

\subsection{The Current Industrial Problem}

As outlined above, HRC seems to offer the potential to not only improve the efficiency and flexibility of modern production processes through better human-robot cooperation and task sharing across the entire manufacturing system, but also to enhance responsiveness to the changing needs of consumer demands and of workers. However, although all of this points toward positive outcomes the current situation is that, as is typical in the development of new technology, our progress in building technical capability is outpacing our knowledge and understanding of its potential impacts on the human user. This does not bode well for industry given that, over the years, we have seen many examples where late or lacking integration of human factors has been detrimental to the operational success of new manufacturing technologies $[15,16]$. It is also not ideal for worker health and wellbeing given that we also know that the design of HRC systems can significantly impact on particular human psychological responses which may also ultimately affect performance, such as trust and acceptance $[17,18]$. It would obviously be preferable if these issues were understood and incorporated in system design.

Safety standards governing industrial robotics are periodically reviewed and updated and now permit closer cooperative human-robot working (to be discussed later) [1]. However, their conventional focus is on setting the technical specifications and guidelines for design and integration. Standards 
rarely, if ever, incorporate any consideration of ethical or psychosocial issues of industrial robotics, even if these factors are likely to impact on the technical safety aspects or system performance. It may also be beneficial, therefore, to more fully understand how industrial HRC will change operator roles and impact on worker performance and wellbeing in order that new standards and revisions can incorporate any relevant design and implementation principles that will ensure that new systems are designed to optimise the operational capability of the human-robot system in its entirety.

\section{THE A4BLUE STUDY}

A4BLUE (Adaptive Automation in Assembly for BLUE collar workers satisfaction in Evolvable context) is a large multi-disciplinary consortium project) which is developing a new generation of sustainable and adaptive assembly work systems that not only incorporate HRC to meet the important efficiency and flexibility requirements / challenges outlined above, but also incorporate fundamental ethical principles and safety standards. Through the development of industrial applications across four use case scenarios (two based in live manufacturing environments and two laboratory based) the project will demonstrate proof of concept for the integration of HRC and other digital manufacturing technologies for enhancing production efficiency and flexibility. The HRC solutions that this work will deliver comprise novel aspects:

- Reflexive HRC to integrate robots and people within shared workspaces and take advantage of each other's skill strengths within evolving conditions

- Adaptive automation and human assistance capabilities to provide reflexive response to changing human, technical and production requirements

- Personalised and context aware interfaces to detect idiosyncratic requirements of individual operators and changing demands

- An integrated rule based model of worker satisfaction to ensure that the adaptive automation and human assistance responses will maintain optimal levels of operator well being

Clearly, these features will support the capability of HRC to enhance efficiency and flexibility as outlined. Previous work has explored new methods for analysing human tasks for transfer to automation [14]. However, the A4BLUE project is novel in that it is also seeking to ensure the integration of safety and ethical principles as a priority. A key activity is to review existing ethical and safety standards in order to identify specifications to which the new HRC solutions must comply, but also to identify gaps - where ethical and safety principles do not yet meet the requirements of cutting edge digital manufacturing technologies. To this end, the project has begun with two foundational activities: identification of 'user' requirements and 'highlevel' requirements.

\subsection{User requirements analysis}

Ethical design needs to be built on usercentredness, as this is the only way to capture and integrate true preferences and requirements from the first-hand accounts of system users / operators. User centred design relies on the user being involved as a co-designer throughout developmental stages and not simply as an 'informant' in later-stage testing, because only they have a valid first-hand understanding of the 'context of use' [19]. To maximise a user-centred design approach and identify aspects of future work system design that might need to be considered in ethics and safety standards, the A4BLUE project began with an exploration of 'multidimensional' user requirements crossing different roles and layers in organisations.

\subsubsection{Method}

A wide range of potential stakeholders and endusers who may be affected by / involved in the implementation of new HRC work systems within organisations in Business, Organisation, Technology or Human user groups were identified. Participants representing each category were recruited from each of the project partners' organisations in the manufacturing and technology development industries.

An online survey was then created to gather opinions about a number of specific design features of future work systems across a number of categories, one of which was Automation and Robotics; questions therefore covered various potential technologies and capabilities, not just HRC. The survey was designed to collect a combination of quantitative data, where participants simply ranked their opinions towards listed items (statements) about individual design features as either essential, desirable, or unnecessary, and qualitative data, for which participants were asked to write freely about the reasons behind their opinions and given the opportunity to provide any other ideas for the design of future assembly work systems. In this way the questionnaire was designed to capture both a measure of people's strength of opinion towards each design feature along with a richer picture of the factors that explain those 
opinions. After the survey web link was administered to recruited participants and fifty responses were received; the online system processed and delivered the data anonymously.

Analysis involved identifying principal user requirements based on the extent to which individual items had been rated as "Essential" and "Desirable". Items were ranked according to combined score frequencies to determine the design features of most priority across the collective data.

\subsubsection{Results}

Across the different design feature categories participants generally showed support for the development of new digital systems, albeit most of the individual technologies were considered desirable rather than essential. This is however this is to be expected to some extent given that many participants were working in industrial technology companies. However, turning to the specific category of Automation and Robotics design features which had a total of twenty-one items, ten items were scored as essential, eight as desirable, and only three were ranked as unnecessary. These are listed below in order of priority ranking.

\section{ESSENTIAL design features}

1. Systems that immediately stop the robot in the event of an accidental collision.

2. System mechanisms that make operators comfortable when collaborating with automation/robots during assembly.

3. System capabilities to adapt the speed of the robot according to the distance or speed of the operator.

4. Robots that move away from the worker in the event of an accidental collision.

5. Robots that work collaboratively and safely with an operator on shared tasks in fenceless environments.

6. Automation / robotics that are controllable by the operators working in the system.

7. Automation / robotics that can change safely by themselves to meet different physical capabilities of the involved operators, such as size differences.

8. Automation / robotics that can self-adapt configuration to an operator's physical characteristics (i.e. height, arm length) to avoid potential ergonomic issues.

9. Capabilities to differentiate between people and other kinds of potential obstacles, and adapt the automation / robot behaviour to suit.

10. System ability to make operators aware of whether or not the safety mechanisms and devices are functioning effectively.

\section{DESIRABLE design features}

1. Automated / robotic functions that will adapt to suit each operator's preferred working methods.

2. Automation / robotics that can change safely to meet varying production demands.

3. Automation / robotics that can change safely on their own to meet different experience capabilities of the involved operators.

4. Automation / robotics that can change safely on their own to meet different environmental conditions like varying light and noise levels.

5. Functionalities to adapt the safety strategy based on the operators preferences and what is happening in the area surrounding the robot.

6. Automation / robots that can adapt speed to correspond with an operator's profile (i.e. expertise, skills, capabilities, preferences, trust level).

7. Robots that notify management about the completion and the status of the task.

8. Robots should work safely alongside or near to an operator but on separate tasks.

These items were designed to address combined issues of safety and personalisation / flexibility. It is of no surprise that the most highly scored item concerns the need for robots to be stopped immediately in the event of an accidental collision, or that other highly scored items deal with requirements for safety-critical functions. However, it is interesting to note that the second highest scored item concerns operator comfort, and that some other highest ranking 'Essential' requirements concern adaptation and personalisation to suit worker characteristics and idiosyncrasies.

It is likely that some of these issues will be related to the psychological responses that impact on performance as discussed, e.g. operator trust and acceptance. Associated system design features may also need to be considered with regard to ethical suitability, such as the acceptability of the personal data acquisition and monitoring that will be needed to create adaptive personalised systems.

This relatively small and simple initial survey gives us an early insight into what should perhaps be considered in future ethics and safety standards for industrial HRC systems. It is reasonable to consider including psychological safety and comfort in addition to technical safety factors because stakeholders and end-users understand the prospect of greater interaction and are not only seeking measures to enhance safety but also their personalised requirements.

\section{2 'High-level' requirements analysis}

In addition to gathering user-level requirements, a 'high-level' requirements analysis has also been 
conducted early in the A4BLUE project, to extract formal requirements that emanate from sources external to stakeholders and users in manufacturing organisations, i.e. from legal, governance and standards frameworks. The aim of this activity was to identify system design requirements but also gaps where current frameworks do not yet cover the technologies - or assemblage of technologies - that are being designed and developed.

\subsubsection{Method}

For this activity the method needed to be a systematic document / literature review to inspect resources that are most relevant to the proposed technologies and features of the A4BLUE systems. Once again this work involved exploring a wide range of technologies and capabilities, not just HRC systems.

The scope of the review covered technical, ethical and human factors / user-centred requirements for a) general industrial work / machinery safety, and b) the specific technical features and technologies (including automation and robotics). To prioritise the A4BLUE research context the review also focused on European Union (EU) manufacturing industry requirements. A review of EU standards was prioritised as these reflect EU directives (law) but more usefully set out technical specifications that are harmonised with international laws and standards. Reviews were prioritised according to relevance of material which was based on applicability to the design of integrated manufacturing systems across four principal design categories: industrial work and machine safety, automation and robotics standards, ergonomics and human factors, and digital systems. Clauses that were considered most pertinent to the design features of new work systems were selected within the assumption that functional characteristics, performance or safety of individual system components will not be changed by their integration in the project and therefore remain in conformity to design standards.

\subsubsection{Results}

The supreme legal governance of industrial machine safety in EU countries comes from the European Machinery Directive 2006/42/EC which has "the dual aim of harmonising the health and safety requirements applicable to machinery on the basis of a high-level of protection of health and safety, while ensuring the free circulation of machinery on the EU market" (European Commission, 2010, p.1). A large number of the standards within this directive, harmonised to align with international standards, may contain small elements that are relevant to HRC but for the purposes of this paper we focus those that are most dedicated to HRC systems in our Automation and Robotics category. Those responsible for developing and updating laws and standards for robotics have the challenge of keeping pace with significant technology advances including the rapid recent expansion of industrial HRC opportunities. On one hand standards need to address new possibilities for adapting conventional hazardous, heavy payload robots into safe HRC systems. On the other hand they also need to consider the increasing potential for applying smaller limited force non-industrial robots, such as healthcare and social robots, in industrial HRC systems.

\section{A-type standard}

The key A-type standard (setting out basic concepts, terminology and principles for design) is adopted from the International Standards Organisation (ISO):

- EN ISO 12100:2010 Safety of machinery - General principles for design Risk assessment and risk reduction is the single A-type standard in the European Machinery Directive, setting out general concepts and fundamental requirements, including a number of risk reduction measures and basic humansystem principles.

\section{C-type standard}

Beneath the type A overarching principals is a two-part C-type standard (application-specific standard) with central relevance to robot design and robot integration. This is also adopted from ISO:

- EN ISO 10218-1:2011 Robots and robotic devices - Safety requirements for industrial robots - Part 1: Robots is the first part of the 10218 standard sets out fundamental technical specifications and guidelines for "safety in the design and construction of the robot" (p.vi). This covers the design of the robot and its protective measures to mitigate basic hazards but does not cover wider issues concerning implementation and integration with other systems, nor does it apply to robots designed for non-industrial applications. As the A4BLUE project will not be designing new robotics but integrating existing commercially available systems, these standards may not be highly relevant unless integration alters performance / functional safety. The human user is addressed in terms of physical ergonomic hazards (due to lighting and controls) and potential consequences (such as incursion, fatigue and stress). However, the interplay between psychological reactions and interactions with robotics is very limited and does not provide 
any practical guidance for how to optimise the human-robot relationship.

- EN ISO 10218-2:2011 Robots and robotic devices - Safety requirements for industrial robots - Part 2: Robot systems and integration is the second part of the 10218 standard which provides a relevant comprehensive set of requirements for the application and implementation of an industrial robot (as specified in part 1) and "the way in which it is installed, programmed, operated, and maintained" (p.v). It guides integrators on how to lessen or eliminate hazards associated with the robot and its integration but not extraneous hazards resulting from applications. With respect to user-centred issues there is very little addressed other than limited technical safety aspects such as human spatial separation and safeguards / incursions.

\section{Technical Specification}

The standards document that is most directly relevant to HRC is a Technical Specification (TS) published by ISO in 2016. A TS is a document created to address matters that are still under technical development or are expected to be addressed in a future international standard. They are published for immediate use and are also used to generate feedback in preparation for a new or revised future standard. In this case a TS has been devised specifically to address the advancing potential for HRC:

- ISO/TS 15066:2016 Robots and robotic devices - Collaborative robots is a TS which was developed to serve as interim guidance for HRC, addressing the more recent technology advances and enablement of closer cooperation and colocation, prior to development / integration of clauses into full standard. The content will be reviewed and incorporated as appropriate into a current revision of ISO 10218. In the meantime it has been adopted in several countries.

\section{British Standard on Robot Ethics}

Finally, a new standard created by the British Standards Institute (BSI) was also considered as highly relevant in that it is pioneering the movement to consider robot ethics:

- BS 8611:2016 Robots and robotic devices. Guide to the ethical design and application of robots and robotic systems is an unusual and innovative standard as it is devoted to supplying ethical principles, which are rarely addressed in national or international standards. It reflects a response to the significant rise in robotics applications and, particularly, in the potential for HRC to become an increasing part of everyday life. As such, the standard sets out general principles and guidelines which apply to different types of robot applications and contexts, e.g. industrial, personal care and medical and, therefore, not just industrial HRC. Nonetheless, the novelty of this standard is that it directly addresses requirements for psychological safety and wellbeing and not just physical / technical safety. Additionally, it includes consideration of new or developing functions that are likely to influence HRC design, including industrial applications, such as personal / performance data management and security and robot adaptation to personalised settings and requirements.

The above review of standards is an extremely brief snapshot of those most relevant to industrial HRC. It does not cover the issues that are currently in standards that are not directly applicable but may be in the future when HRC systems comprise more advanced functions, such as data security and privacy. However, it indicates the current state is that existing specifications and guidelines used by industry are still almost entirely focused on technical and system safety. This is very understandable given that the convention has been to segregate robots into wholly technical areas in hybrid manufacturing systems and, therefore, it is only necessary to consider human involvement in relation to controls and contraventions. However, the current tide of increasingly closer and interactive $\mathrm{HRC}$ is going to require more direct attention to other 'softer' human issues if they are important to system safety and performance. This is where the topic of robot ethics becomes relevant; whereas it has not been a valid consideration in traditional manufacturing processes it is now the case that safety standards should now begin to consider how systems will impact on users both physically and psychologically. The publication of BS 8611 provides a positive and forward-thinking set of guidelines but its generic approach does not satisfy the needs of new industrial systems which will entail distinct production and operator requirements.

\section{CONCLUSION}

The work described in this paper to identify requirements for $\mathrm{HRC}$ systems design has been limited due to the infancy of the project. The userlevel analysis shows that stakeholders and end-users of HRC systems appreciate that future systems will involve greater interaction and that there is a need for not only safety but personalised responses. The user requirements survey will be extended at a later 
stage of the project in order to gather opinions from a wider and more international sample of stakeholders and user groups; this will enable statistical analysis for a more robust set of findings.

The high-level requirements review has demonstrated that, currently, there is a restricted focus on technical system safety which has been perfectly adequate for a wholly technical system but is now becoming an outdated limitation with increasing levels of HRC in industrial systems. The high-level analysis will also be repeated at a later stage of the project in order to check developments and update current results.

Together these two levels of analysis have captured an initial identification of requirements which sets a foundation for better understanding what is likely to be needed in forthcoming ethical and safety standards. These requirements are being used to inform the design and definition of the project's use case systems in which new HRC systems will be built. Subsequent work in the project will then provide updated and confirmatory analysis to define these requirements more effusively.

Figure 1. Initial requirements identification will lead to final requirements definition.

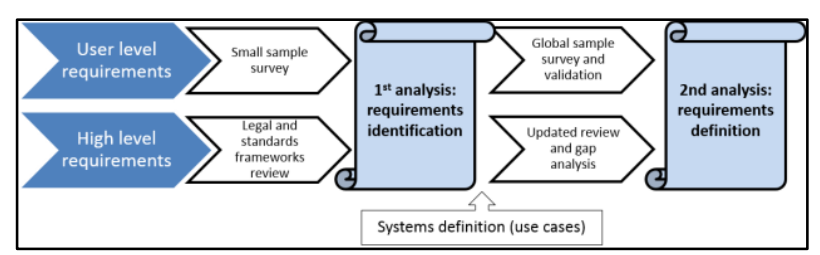

There is one final important point to note. Robot ethics is becoming an increasingly popular topic of investigation and discussion in the general public but it currently pays little attention to industrial robotics. The work described in this paper focused on identifying the ethical and user-centred requirements in current safety standards covering HRC and found gaps relating to consideration of human psychological safety and performance. However, it also found that in the other direction, within the rising topic of robot ethics, there is very little interest in industrial HRC applications. Perhaps this is another consequence of traditional attitudes which assume industrial robots are still heavy and hazardous and segregated, or because the industrial context is considered to be self-contained and detached. It could also be that the standards development communities responsible for industrial safety are equally detached and do not engage with any 'soft' ethical issues about operator well-being because those are not considered relevant to their traditional prioritisation of technical safety. Nonetheless, the preliminary findings presented in this paper suggest that industrial robot ethics is an issue that needs to be explored and understood with rising HRC in manufacturing, and this presents a potential candidate for new safety standards.

\section{ACKNOWLEDGEMENTS}

The work described in this paper was conducted as part of the A4BLUE research project (www.a4blue.eu) funded by the European Commission's Horizon 2020 programme. The authors would like to thank the EC for that support, and the individual partners in the project consortium for their assistance in organising data collection.

\section{REFERENCES}

[1] Walton, M., Webb, P. \& Poad, M. (2011) Applying a concept for robot-human cooperation to aerospace equipping processes, SAE International

[2] De Krüger, J., Lien, T.K. \& Verl, A. (2009). Cooperation of human and machines in assembly lines. CIRP Annals - Manufacturing Technology, 58, 628-646

[3] International Federation of Robotics (IFR), 2017. The Impact of Robots on Productivity, Employment and Jobs. A Positioning paper by the International Federation of Robotics, April 2017.

[4] Bogue, R. (2017). Robots that interact with humans: a review of safety technologies and standards. Industrial Robot: An International Journal, 44(4).

[5] Matthias, B., Kock, S., Jerregard, H., Kallman, M., Lundberg, I., and Mellander, R. Safety of collaborative industrial robots: Certification possibilities for a collaborative assembly robot concept. In Proc. ISAM '11 (2011), 1-6

[6] Hedelind, M. \& Kock, S. (2011). Requirements on flexible robot systems for small parts assembly, a case study. Proceedings of the International Symposium on Assembly and Manufacturing, 25-27 May, Tampere, Finland, 2011.

[7] Michalos, G., Makris, S., Spiliotopoulos, J., Misios, I., Tsarouchi, P., \& Chryssolouris, G. (2014). ROBO-PARTNER: Seamless HumanRobot cooperation for intelligent, flexible and safe operations in the assembly factories of the future. Procedia CIRP, 23, 71-76.

[8] Unhelkar, V.V., Siu, H.C., Shah, J.A.: Comparative performance of human and mobile robotic assistants in collaborative fetch-and-deliver tasks. In: Proc. 2014 ACM/IEEE Int. Conf. Human-robot Interact-HRI'14, pp. 82-89 (2014) 
[9] McCarthy, I. P. (2004). Special issue editorial: the what, why and how of mass customization. Production Planning \& Control, 15(4), 347351.

[10] Pawar, V.M., Law, J., and Maple, C. (2016). Manufacturing robotics - the next robotic industrial revolution. Technical report, UK Robotics and Autonomous Systems Network. http://hamlyn.doc.ic.ac.uk/ukras/sites/default/files/UK_RAS_wp_manufact uring_web.pdf

[11] Favell, A., Feldblum, M., \& Smith, M. P. (2007). The human face of global mobility: A research agenda. Society, 44(2), 15-25.

[12] Stedmon, A. W., Howells, H., Wilson, J. R., \& Dianat, I. (2012). Ergonomics/human factors needs of an ageing workforce in the manufacturing sector. Health promotion perspectives, 2(2), 112.

[13] Pitts, D. W., \& Recascino Wise, L. (2010). Workforce diversity in the new millennium: Prospects for research. Review of public personnel administration, 30(1), 44-69.

[14] Wang, X. V., Kemény, Z., Váncza, J., \& Wang, L. (2017). Human-robot collaborative assembly in cyber-physical production: Classification framework and implementation. CIRP Annals-Manufacturing Technology.

[15] Chung, C. A. (1996). Human issues influencing the successful implementation of advanced manufacturing technology. Journal of Engineering and Technology Management, 13(3-4), 283-299.

[16] Lewis, M. W., \& Boyer, K. K. (2002). Factors impacting AMT implementation: an integrative and controlled study. Journal of Engineering and Technology Management, 19(2), 111-130.10.

[17] Charalambous, G., Fletcher, S., \& Webb, P. (2016). The development of a scale to evaluate trust in industrial human-robot collaboration. International Journal of Social Robotics, 8(2), 193-209.

[18] Charalambous, G., Fletcher, S., \& Webb, P. (2015). Identifying the key organisational human factors for introducing human-robot collaboration in industry: an exploratory study. The International Journal of Advanced Manufacturing Technology, 81(9-12), 21432155.

[19] Gulliksen, J., Göransson, B., Boivie, I., Blomkvist, S., Persson, J., \& Cajander, A. (2003). Key principles for user-centred systems design. Behaviour and Information Technology, 22(6), 397-409.

[20] European Commission (2010). Guide to application of the Machinery Directive 2006/42/EC (2nd. Ed); accessed 15/09/17 from:

http://ec.europa.eu/enterprise/sectors/mechani cal/files/machinery/guideappl-2006-42-ec2nd-201006_en.pdf 
2017-12-31

\section{Putting people and robots together in manufacturing: are we ready?}

Fletcher, Sarah R.

Unknown

Fletcher SR, Johnson TL, Larreina J. Putting people and robots together in manufacturing: are we ready? International Conference on Robot Ethics and Safety Standards, 2017, 20-21 October 2017, Lisbon, Portugal

https://www.icress2017.org/

Downloaded from Cranfield Library Services E-Repository 